\title{
Improvement of high temperature fault problem of Wind power inverter based on TRIZ
}

\author{
LinLin Sun ${ }^{1}$; Yang Liu $^{2}$; JiaYu Xie ${ }^{1}$; Rui Cao ${ }^{1}$ \\ ${ }^{1}$ Jilin University, College of Mechanical and Aerospace Engineering, Changchun 130022, China \\ ${ }^{2}$ Datang Jilin Power Generation Co, Ltd.130022, China
}

\begin{abstract}
The research content of this paper is to improve the high temperature fault problem of the wind power inverter and optimize the processing process of the high temperature fault problem. In order to solve the high temperature fault problem, the water-cooling system of inverter is analyzed in detail by using the function analysis, cause and effect analysis and object field analysis in the invention problem solving theory (TRIZ theory). At the same time, combined with the invention principle of TRIZ theory and the standard solution, seven different solutions are finally obtained. Based on the ECRS principle, the general process of high temperature fault problem treatment is simplified through continuous optimization and improvement.
\end{abstract}

\section{Introduction}

As an important part of the wind power plant connected with the power grid, inverter has considerable social benefits and a wide range of applications. However, the inverter will generate a lot of heat in the process of operation, which will lead to frequent high temperature failures, and even cause serious accidents and economic consequences.

At home and abroad, there are many research achievements on inverter heat dissipation. Foreign research on liquid cooling related technologies started very early, and related technologies are becoming mature. Although China started relatively late and still lags behind the international advanced level in terms of core components, many new technological achievements have emerged in recent years with rapid development and good momentum. At present, foreign research has been carried out on the material transformation of cold plate passage. Porous material heat sink is used as the material to improve its heat transfer efficiency [1]. The fin of the fluid passage is created, and the radiator is improved obviously and intuitively [2]. By modifying the internal structure of the cold plate, the triangular fluid path is obtained to improve its heat transfer effect [3]. In recent years, a new equivalent thermal circuit diagram considering NTC thermistor in IGBT module has been proposed in China [4]. The cooling device of the megawatt power device IGBT was optimized, and the cold plate structure with superior comprehensive performance was obtained [5].

Although the research on inverter is relatively mature, there are few inverter heat dissipation achievements and flow optimization in the field of wind power generation. Therefore, based on the TRIZ theory and ECRS principle, this paper will improve the high temperature fault and the cooling system of the inverter and optimize the process of fault handling.

\section{Current situation and problem analysis of inverter and water cooling system}

\subsection{Inverter with water cooling system}

\subsubsection{Net side inverter}

The grid-side inverter module is located in the variableflow control cabinet, and the ceramic substrate of its internal IGBT module is connected with the watercooled substrate via the heat-conducting silica gel. There is a wind cooling system in the converter control cabinet, which can create a wind cooling cycle in the converter control cabinet to avoid local overheating of the inverter.

\subsubsection{Water circulation cooling system}

(1) Cooling control cabinet

The water circulation cooling system is located in the cooling control cabinet. The cooling control cabinet consists of two parts: the circulating cooling system and the measuring control system. The schematic diagram of the water circulation cooling system is shown in FIG. 1.

\footnotetext{
Corresponding author: slfdly6009@163.com
} 


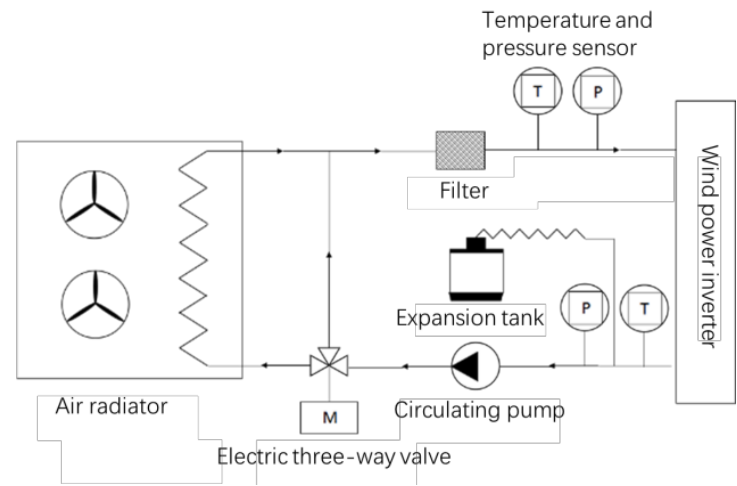

Fig. 1. Schematic diagram of water circulation cooling system

\section{(2) PLC}

The core of the measurement control system in the cooling control cabinet is PLC, which monitors the cooling circulation system in real time.

\section{(3) Cooling fluid}

The coolant is a mixture of purified water, ethylene glycol and preservatives.

(4) Water cold plate pipe

The water enters the water-cooled substrate from the inlet pipe, then flows through the water-cooled pipe, and then out of the outlet pipe, where the water passes right across the bottom of the IGBT.

(5) Water cooling system parameters

See Table 1. for the parameter setting of water cooling system:

Table 1. Water cooling system parameter setting

\begin{tabular}{ccc}
\hline $\begin{array}{c}\text { The serial } \\
\text { number }\end{array}$ & Project & $\begin{array}{c}\text { Normal } \\
\text { values }\end{array}$ \\
\hline 1 & System static pressure & $2.0 \mathrm{bar}$ \\
2 & Dynamic pressure of inlet valve & $2.1 \mathrm{bar}$ \\
3 & Exit valve dynamic pressure & $1.7 \mathrm{bar}$ \\
4 & Flow & $290 \mathrm{~L} / \mathrm{min}$ \\
5 & Inlet valve temperature & $<45^{\circ} \mathrm{C}$ \\
6 & Temperature difference of inlet & $<5.5^{\circ} \mathrm{C}$ \\
\hline
\end{tabular}

\subsection{Existing problems}

With the continuous increase of the ambient temperature, when the wind power generating unit reaches full capacity in the windy weather, "grid side inverter temperature fault" is frequently reported. Table2. and Table3. are the fault file data of different locations(Both Bayin and Guantin are local place names), as shown below:

Table 2. Bayin scene field failure file data (Temperature of the unit/ $\mathrm{C}^{\circ}$ )

\begin{tabular}{clllll}
\hline $\begin{array}{c}\text { Machine } \\
\text { number }\end{array}$ & $\begin{array}{l}\text { Ambient } \\
\text { temperature }\end{array}$ & $\begin{array}{l}\text { Inlet valve } \\
\text { temperature }\end{array}$ & $\begin{array}{l}\text { Outlet valve } \\
\text { temperature }\end{array}$ & $\begin{array}{l}\text { Inverter } \\
\text { temperature }\end{array}$ & Fault time \\
\hline $38 \#$ & 24.5 & 40.5 & 42.9 & 68 & $13: 09$ \\
$51 \#$ & 32.6 & 38.1 & 40.1 & 68 & $14: 15$ \\
$63 \#$ & 23.8 & 37.1 & 39.1 & 68 & $15: 51$ \\
$89 \#$ & 24.3 & 37.3 & 39.4 & 68 & $21: 54$ \\
$89 \#$ & 23.8 & 37.7 & 39.9 & 68 & $23: 43$ \\
$95 \#$ & 24.7 & 38.3 & 40.4 & 68 & $15: 12$ \\
$95 \#$ & 25.0 & 38.1 & 40.1 & 68 & $16: 18$ \\
\hline
\end{tabular}

Table 3. Guantin scene field failure file data (Temperature of the unit/ $\mathrm{C}^{\circ}$ )

\begin{tabular}{clllll}
\hline $\begin{array}{c}\text { Machine } \\
\text { number }\end{array}$ & $\begin{array}{l}\text { Ambient } \\
\text { temperature }\end{array}$ & $\begin{array}{l}\text { Inlet valve } \\
\text { temperature }\end{array}$ & $\begin{array}{l}\text { Outlet valve } \\
\text { temperature }\end{array}$ & $\begin{array}{l}\text { Inverter } \\
\text { temperature }\end{array}$ & Fault time \\
\hline $13 \#$ & 34.0 & 40.9 & 42.5 & 68 & $13: 10$ \\
$15 \#$ & 35.6 & 40.6 & 42.3 & 68 & $15: 11$ \\
$16 \#$ & 32.3 & 38.3 & 40.0 & 68 & $12: 16$ \\
$27 \#$ & 32.3 & 40.9 & 42.9 & 68 & $12: 10$ \\
$3 \#$ & 35.3 & 40.5 & 42.2 & 68 & $15: 10$ \\
$35 \#$ & 34.0 & 40.4 & 42.2 & 68 & $13: 05$ \\
$87 \#$ & 35.2 & 42.5 & 43.7 & 68 & $15: 17$
\end{tabular}

According to the above table, the temperature difference between the on-site inverter temperature and the outlet valve temperature is about $26{ }^{\circ} \mathrm{C}$, which is obviously too high, indicating that the heat dissipation efficiency between the inverter IGBT module and the water cooling system is not enough. It can be seen that the water cooling system needs to be improved, and the specific analysis is as follows:

The difference between Bayin scene and Guanting scene fault file data is the temperature difference between the inlet valve water temperature and the ambient temperature.The temperature difference on site 
in Guanting is about $7{ }^{\circ} \mathrm{C}$, which is $2{ }^{\circ} \mathrm{C}$ higher than the $5{ }^{\circ} \mathrm{C}$ required by the design, indicating that there is still a certain lack of radiator cooling capacity, which did not fully meet the requirements of the radiator design at that time.The temperature difference in Bayin is about 14 degrees Celsius, more than twice the original radiator design requirements.

There are two main reasons for this large discrepancy: One is the natural wind,At that time, the wind direction of Bayin site was just opposite to the blowing direction of radiator fan, so the radiator cooling effect was reduced.The wind direction at the time of the unit failure in guanting was exactly 90 degrees to the blowing direction of the radiator fan, so it had no significant impact on the radiator's cooling effect; second, the radiator did have insufficient cooling capacity.

To verify this, the measured data from Bayin site are shown in Table 4 (the wind direction is exactly 90 degrees from the direction of the radiator fan at the time of measurement).

From the measured data can be seen that the temperature difference between the inlet valve water temperature and the ambient temperature is about 9 degrees Celsius, compared with the original 14 degrees Celsius has a great improvement, so it can be explained that the wind direction of the radiator heat dissipation will also have a great impact.

Table 4. Measurement data of Bayin site (Temperature of the unit/ $\mathrm{C}^{\circ}$ )

\begin{tabular}{llcccc}
\hline $\begin{array}{l}\text { Machine } \\
\text { number }\end{array}$ & $\begin{array}{l}\text { Ambient } \\
\text { temperature }\end{array}$ & $\begin{array}{l}\text { Inlet valve } \\
\text { temperature }\end{array}$ & $\begin{array}{l}\text { Outlet valve } \\
\text { temperature }\end{array}$ & $\begin{array}{l}\text { Inverter } \\
\text { temperature }\end{array}$ & $\begin{array}{l}\text { Radiator } \\
\text { temperature range }\end{array}$ \\
\hline $1 \#$ & 25.5 & 38.5 & 39.7 & 63 & $30-37$ \\
$2 \#$ & 25.6 & 33.6 & 35.1 & 61 & $26-34$ \\
$10 \#$ & 26.1 & 36.5 & 38.1 & 61 & $29-36$ \\
$19 \#$ & 26.5 & 34.1 & 35.8 & 59 & $29-34$ \\
$8 \#$ & 26.7 & 34.3 & 35.5 & 60 & $27-34$ \\
$25 \#$ & 26.8 & 35.0 & 36.1 & 63 & $24-33$ \\
\hline
\end{tabular}

\section{Analysis and improvement of high temperature fault based on TRIZ}

\subsubsection{Building functional models}

Combining the interaction matrix and the function 3.1. Functional analysis list of components, the function structure model is established, as shown in the fig 2:

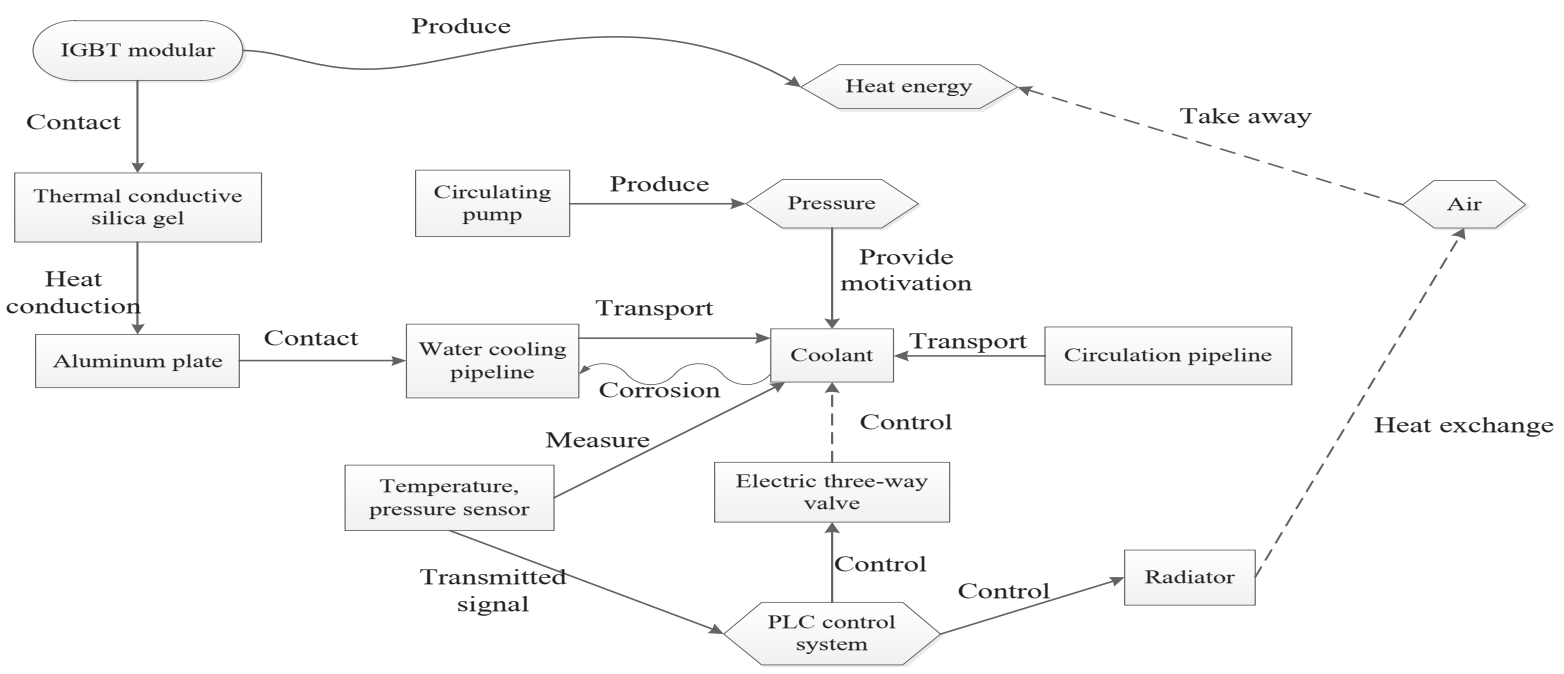

Fig. 2. Functional structure model

\subsubsection{Field analysis and solution}

(1) For the functional analysis of water-cooling pipes and coolants, $\mathrm{S} 1$ = water-cooling pipes, $\mathrm{S} 2=$ coolants.
The function model is represented by symbol system as shown in Fig 3: 


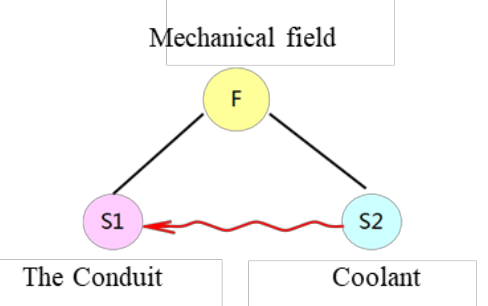

Fig. 3. Object - field model of water - cooled pipeline and coolant

Using standard solution 1.2.2 to introduce improved S1 or (and) S2 to eliminate harmful effects, that is, the existing system is complete, harmful and useful effects exist at the same time, the third substance S3 can be introduced between substances to eliminate harmful effects, S3 is the deformation of existing substances.

Scheme 1 is proposed: : S3 is the deformation of S2 coolant. The existing coolant is composed of glycol and purified water, which is corrosive and easy to cause harmful effects on the pipeline. The introduction of 1,2propanediol, which is non corrosive and easy to degrade, can reduce the corrosion effect of coolant on metal pipes. (2) For the functional analysis of the electric three-way valve and coolant, $\mathrm{S} 1=$ coolant, $\mathrm{S} 2$ = electric three-way valve. The function model is represented by the symbol system as shown in Fig 4.

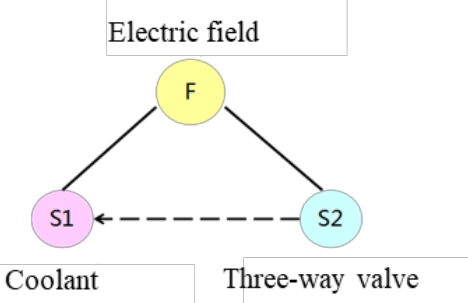

Fig. 4. object field model of three-way valve and coolant

Using more controllable field by standard solution 2.2.1: the controllability of the object field model is insufficient. The field that is easy to control can be used

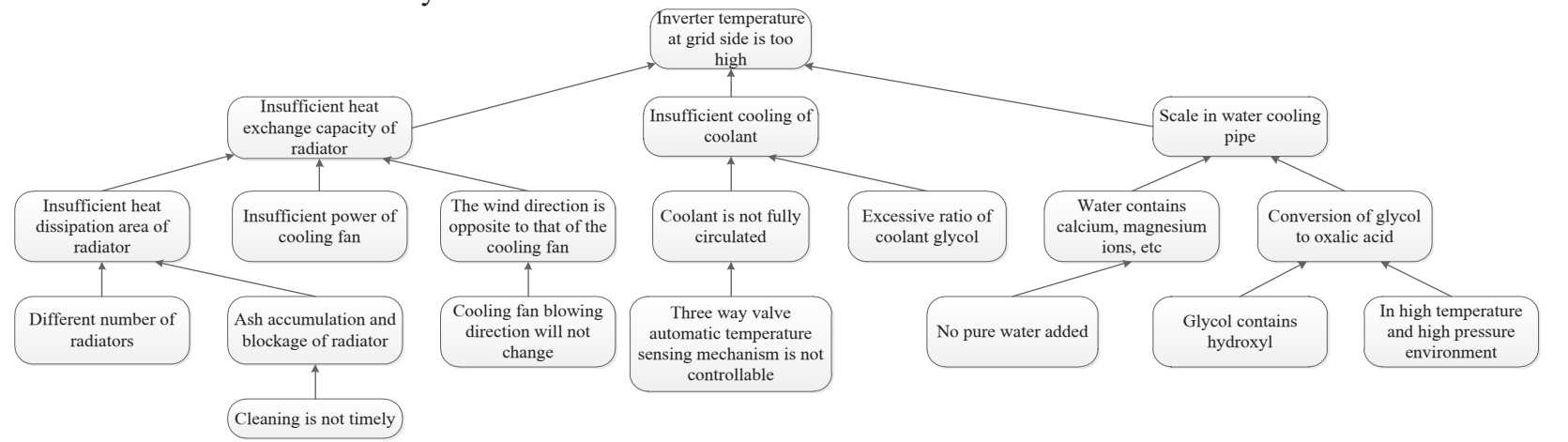

Fig. 6. Cause and effect analysis chart

Considering the practical problems such as optimization cost and feasibility, four root causes are summarized: (1) the radiator is blocked by ash deposition; (2) the wind direction of the cooling fan does not change; (3) the automatic temperature sensing mechanism of the threeway valve is not controllable; (4) the content of glycol in the coolant is not accurate. Through 40 principles of to replace the field that is difficult to control (from difficult to easy, it can be arranged as mechanical field, thermal field, chemical field, electric field and magnetic field), so as to improve the controllability.

Scheme 2 is proposed: : magnetic control valve is used instead of electric three-way valve to achieve more accurate control.

(3) For the functional analysis of cooling fan and air, S1 = air, $\mathrm{S} 2$ = radiator. The function model is represented by symbol system as shown in Fig 5:

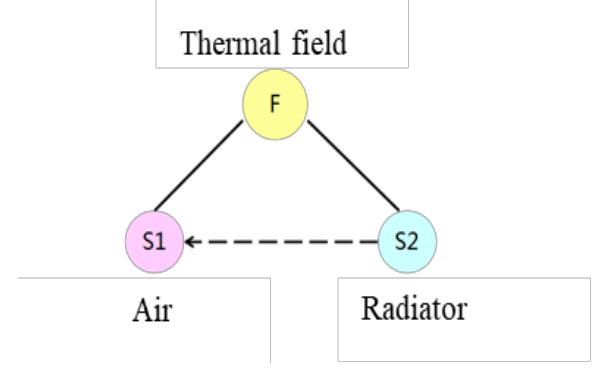

Fig. 5. object field model of cooling fan and air

Use standard solution 2.2.3 to use substances with capillary and porous structure: change the structure of substances to become substances with capillary or porous structure, and let gas or liquid pass through these substances with capillary or porous structure, so as to obtain the enhancement of system function effect.

Scheme 3 is proposed: change the fins in the radiator into fins with staggered hole structure, increase the heat exchange area, so as to improve the heat exchange efficiency of the radiator.

\subsection{Causal analysis}

\subsubsection{Causal analysis diagram}

The causal analysis diagram is shown in fig 6: 
more manpower and time cost, as well as equipment shutdown operation. Therefore, there is a technical contradiction. The improved parameter is the harmful factor of the object, and the deteriorated parameter is the time loss. The principles of invention 35, change of physical or chemical parameters, 18, mechanical vibration, 34 abandonment and regeneration are applied. Inspired by the change principle of physical or chemical parameters, scheme 4 is proposed: the radiator with selfcleaning function is obtained by coating a layer of nano $\mathrm{TiO}$ film on the surface of the radiator.

(2) When the wind direction is opposite to the blowing direction of the cooling fan, the heat transfer capacity of the radiator will be affected, and the wind direction is uncontrollable. Therefore, it can be considered to change the direction of the cooling fan, so that the radiator can be adjusted to rotate 90 degrees. But adding a device that can adjust the direction of radiator increases the complexity of the device. At this time, the parameters of technical contradiction improvement are adaptability and universality, and the parameters of deterioration are the complexity of the system. According to the contradiction matrix, the principle of invention is 15 dynamic characteristic principle, 34 abandonment and regeneration principle, 1 division principle, 16 unreachable or excessive action principle.

According to the principle of dynamic characteristics and the principle of partition, scheme 5: improve the dispersion degree of air radiator and water cooling system, and make it supported by the load-bearing device which can adjust the direction. And a wind direction sensor is set, which can make the device adjust the angle with the change of wind direction.

(3) The automatic temperature sensing mechanism in the three-way valve regulates the amount of internal and external circulation of coolant according to different water temperature, so it can not control the internal and external circulation of coolant arbitrarily according to the actual needs. Want to get more accurate and convenient control, but at the same time change its automation. That is to say, the improved parameter is the convenience of the operation process, and the deteriorated parameter is the degree of automation. The invention principle is as follows: 1. Division principle, 34. Abandonment and regeneration principle, 12. Equipotential principle, 3. Local mass principle.

By using the segmentation principle, scheme 6 is obtained: setting two modes: automatic mode and manual mode. In the manual mode, the coolant flow rate of inner and outer circulation can be determined according to the actual situation, which is more convenient to control.
(4) When the coolant is used for antifreeze, it is required to increase the content of glycol in the coolant; when it is necessary to play the cooling and heat dissipation role of the coolant, it is necessary to reduce the content of glycol in the coolant. There is a physical contradiction. Using time separation, we can get scheme 7: in the period of frequent high temperature failure, we can take out about $10 \%$ of the coolant in the water cooling system, and then add $10 \%$ of the pure water into the coolant. In winter, about $10 \%$ of the coolant in the water cooling system can be taken out, and then $10 \%$ glycol can be added to the coolant for replenishment.

\section{Process optimization of high temperature fault treatment based on ECRs principle}

\subsection{High temperature fault}

\subsubsection{Fault description}

Fan fault is usually found by fan alarm and artificial fan data analysis.

Table 5. Explanation of inverter high temperature fault

\begin{tabular}{cc}
\hline Category & Explain \\
\hline Fault name & $\begin{array}{c}\text { High temperature of grid } \\
\text { side inverter }\end{array}$ \\
Fault description & Temperature over limit \\
Failure time & $100 \mathrm{~ms}$ \\
Fault set value & $67^{\circ} \mathrm{C}$ \\
Reset value & $57^{\circ} \mathrm{C}$ \\
\hline
\end{tabular}

\subsubsection{Cooling system related faults}

Although the small problems of each component of the cooling system are less than the degree of failure, they will also affect the heat exchange efficiency, thus affecting the temperature of the inverter. There are 13 related problems, such as temperature fault of control cabinet, fan fault of water cooling system, pressure fault of inlet and outlet valve, temperature fault of inlet and outlet valve, low pressure fault of inlet valve, warning of water cooling fan, etc.

\subsection{General processing flow of inverter high temperature fault}

The general processing flow chart of inverter high temperature fault is shown in Fig 7: 


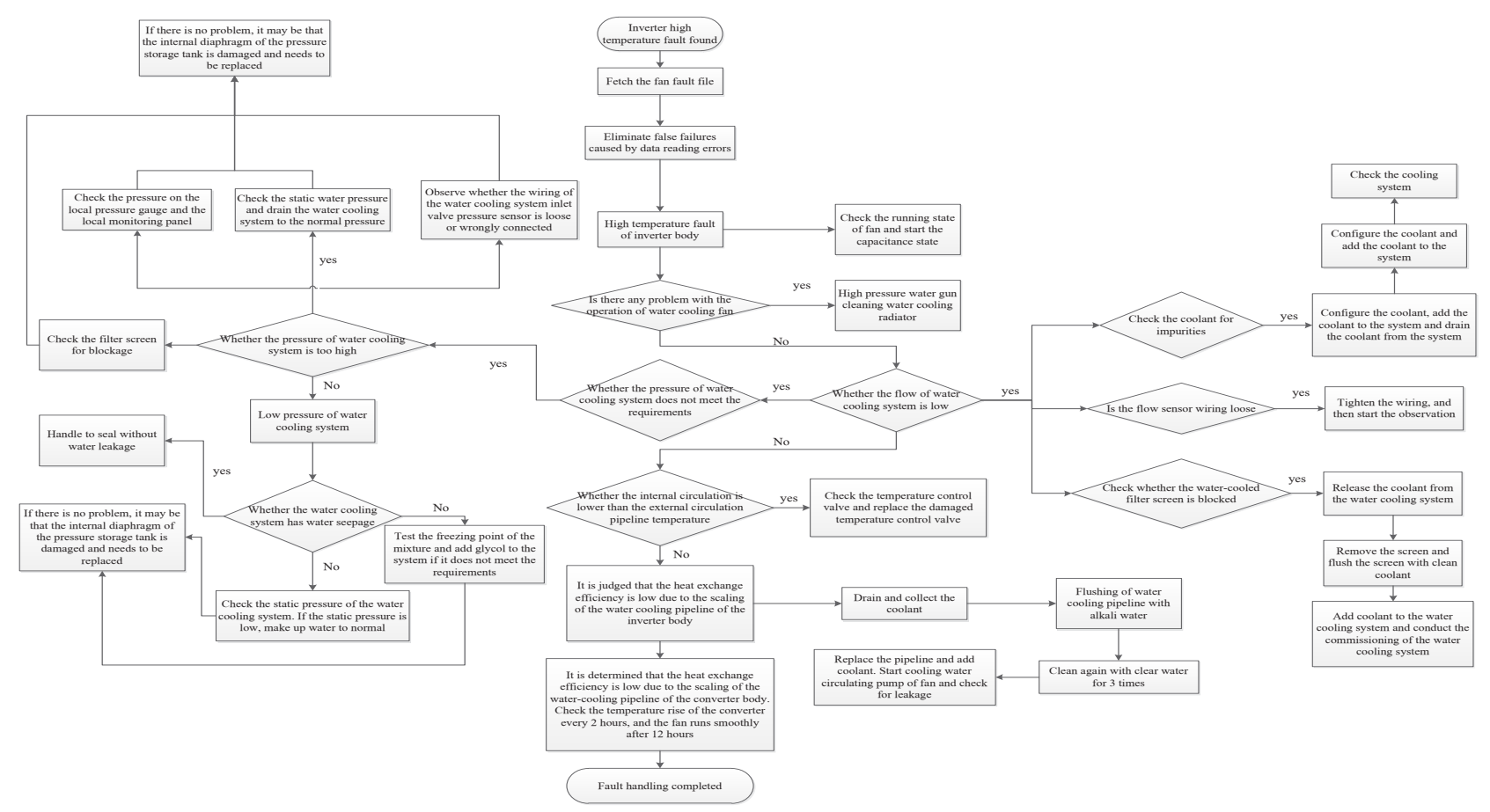

Fig.7. general processing flow chart of inverter high temperature fault

\subsection{Use ECRS principle to optimize the process}

\subsubsection{Cancel}

(1) When there is a problem in the air cooling system of the converter control cabinet, the possibility of high temperature fault reported by the converter module and the controller module at the motor side is greatly increased. If only the inverter module reports high temperature fault, the temperature of the controller and converter is within the normal range, and there is no sudden rising trend of temperature, the step of checking the cooling fan of the control cabinet can be cancelled. the two steps of checking whether the flow sensor wiring is loose and whether the temperature sensor is loose can also be cancelled.

( 2 ) Because the staff will regularly check the operation of the cooling fan and clean it. Therefore, if a high temperature fault occurs within a short time after the inspection and cleaning time, it can be considered to cancel the inspection of this step.

( 3 ) The real-time monitoring data of the main controller shows that the pressure of the water-cooling system is on the high side, while the pressure on the local pressure gauge and the monitoring panel is normal, which indicates that the pressure sensor of the inlet valve has problems, transmits error signals, and the pressure of the water-cooling system is normal. It shows that this detection step has little correlation with high temperature fault, and can be cancelled. Similarly,

(4) When the pressure of the water cooling system is low, it may be due to the ice phenomenon of the mixture in the low temperature environment. Therefore, when the pressure of the water cooling system is low, there will be the ice point value of the test coolant. If it does not meet the requirements, add glycol to the coolant in the water cooling system, so as to reduce the possibility of ice formation at low temperature. But because this is to deal with the inverter high temperature fault, the ambient temperature of high temperature fault will not be low, so this step is canceled.

\subsubsection{Merge}

The low flow of water-cooling system is caused by abnormal system pressure or clogged filter screen. The abnormal system pressure may also be caused by clogged filter screen. Therefore, there are two times to check whether the filter screen is clogged in the process. Therefore, the filter screen shall be merged and replaced until the low flow of water-cooling system is found.

\subsubsection{Rearrangement}

According to 30 seconds data before and after the fault to eliminate the false fault caused by data reading error, at the same time, judge whether the flow of watercooling system is low and whether the pressure of watercooling system meets the requirements. Comparing the flow of water-cooling system with the normal value in advance to eliminate the false fault caused by data reading error, it is more convenient and time-saving to avoid multiple data viewing at different times.

\subsubsection{Simplify}

(1) When the filter screen of the water-cooling system is blocked, the filter screen needs to be removed from the water-cooling system for cleaning. Two ball valves can 
be installed on both sides of the filter screen. When cleaning is needed, the ball valve can be closed, so it is not necessary to discharge all the coolant to supplement these two steps, which simplifies the operation, saves a lot of time, and improves the cleaning efficiency of the filter screen.

(2) When the scale accumulation in the water-cooling pipeline is washed with the alkali water at the ratio, the reverse flushing method can be adopted, that is, the single-module outlet pipe of the original inverter is connected with the outlet pipe of the water-adding device, and the inlet pipe of the original single-module inverter is connected with the water tank of the wateradding device, so the flushing efficiency will be higher and the flushing time can be shortened.

\subsubsection{The optimized process}

The processing flow of high temperature fault of the optimized inverter is shown in Fig 8:

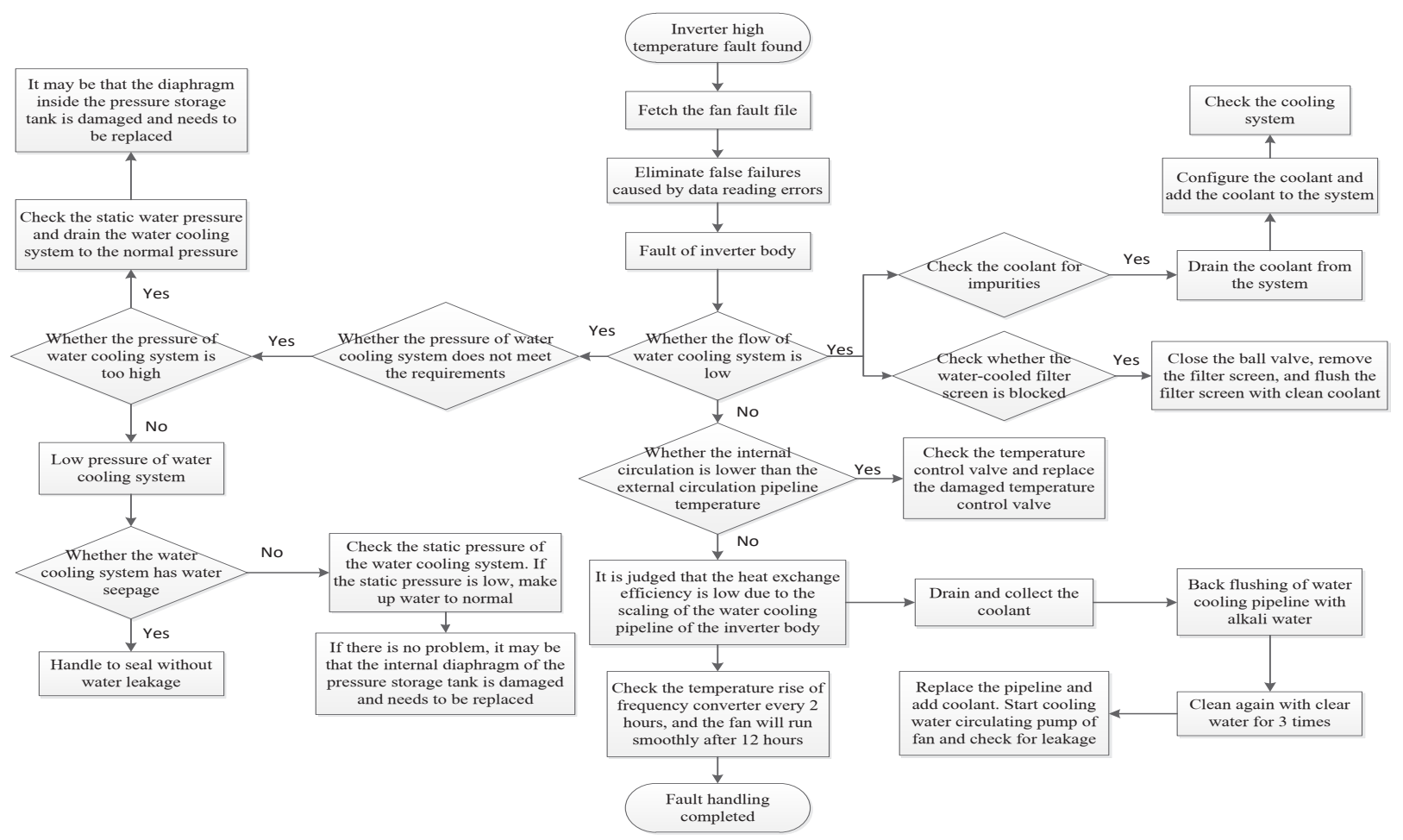

Fig.8. Processing flow of high temperature fault of inverter after optimization

\section{Conclusions}

By using TRIZ theory, this paper analyzes the high temperature fault of wind power inverter and the improvement of cooling system, and puts forward a feasible scheme. Combined with the functional analysis, the cooling system is introduced into the non corrosive and easy to degrade 1, There are three schemes: 2propanediol, magnetic control valve instead of electric three-way valve, changing the radiator fins into fins with staggered hole structure; combined with cause and effect analysis, four schemes are obtained: changing the surface material of radiator fins, designing a wind direction sensor, setting manual and automatic modes, changing the content of glycol in the coolant timely. It shows that TRIZ theory provides people with an efficient and innovative way of thinking and plays a strong role in the actual industrial production process. At the same time, through the principle of ECRs, the process of troubleshooting is optimized, the processing cost and time are reduced, and the production efficiency is increased.

\section{References}

1. Lu G, Zhao J, Lin L, et al. A new scheme for reducing pressure drop and thermal resistance simultaneously in microchannel heat sinks with wavy porous fins[J]. International Journal of Heat and Mass Transfer, 111: 1071-1078.(2017)

2. Kanargi O B, Lee P S, Yap C. A numerical and experimental investigation of heattransfer and fluid flow characteristics of a cross-connected alternating converging-diverging channel heat $\operatorname{sink}[\mathrm{J}]$. International Journal of Heat and Mass Transfer ,106:449-464. (2016)

3. Wong $\mathrm{K}$, Lee $\mathrm{J}$. Investigation of thermal preformance of microchannel heat sink with triangle ribs in the transverse microchambers[J]. International Communications in Heat and Mass Transfer, 65: 103-110. (2015)

4. HongLu Zhao,YongYuan Zhu,Yin Zhang.Heat dissipation design of high power inverter[J].Electrical technology, 19(08):149-156. (2018)

5. ShuYing Tang. Cold plate structure optimization design of megawatt wind power converter[D]. Zhejiang University, (2016). 